\title{
Modeling the shear-dependent viscosity of nonionically stabilized waterborne dispersions
}

\author{
Silfredo J. Bohórquez ${ }^{1,2}$. José M. Asua ${ }^{1}$
}

Received: 15 November 2021 / Revised: 12 January 2022 / Accepted: 12 January 2022 / Published online: 22 February 2022

(c) The Author(s) 2022

\begin{abstract}
The performance of a rheological model that accounts for the effect of the volume fraction, the particle size distribution, and shear rate on the viscosity of water-borne dispersions stabilized with conventional nonionic surfactants and polymeric stabilizers is assessed. The model that contains three parameters fitted well the experimental data. The parameters were independent of the volume fraction, the particle size distribution, and the shear rate. Furthermore, two of them were not affected by the surfactant type and concentration, and temperature. The other parameter increased with the surfactant molecular weight and surface covering, but decreased with increasing temperature.
\end{abstract}

Keywords Rheological model $\cdot$ Polymeric surfactants $\cdot$ Waterborne dispersion $\cdot$ High-solid content latexes

\section{Introduction}

Waterborne polymeric dispersions (latexes) commercially available have in most of the cases solid contents greater than $50 \mathrm{wt} \%$ [1]. Polymeric dispersions with high solid content are very attractive from an industrial point of view because they maximize reactor capacity, minimize transport costs, and enhance flexibility in product formulation [2]. Solid content has a strong effect on the rheology of the dispersion, because viscosity tremendously increases when the solid content approaches the maximum packing factor. On the other hand, most latexes are shear-thinning (pseudoplastic), namely, that their viscosity decreased as the shear rate increases. The rheology plays a critical role in both the production and application of latexes. The latexes are commonly produced in semicontinous operations, where the production rate is determined by the heat removal capability, which in turn, strongly depends on the rheology of the latex

José M. Asua

jm.asua@ehu.eus

1 POLYMAT, The University of the Basque Country UPV/ EHU, Centro Joxe Maria Korta Avenida Tolosa 72, Donostia-San Sebastian 20018, Spain

2 Allnex Netherlands BV, Synthesebaan 1, PO Box 79, 4600 AB Bergen op Zoom, The Netherlands because the heat transfer coefficient substantially decreases as viscosity near the reactor wall increases. In this regard, it is worth pointing out that the pseudo-plastic behavior of the latexes results in a higher viscosity near the reactor wall where the shear rate is low. High viscosity can also yield poor mixing that may lead to local variations of the concentrations of the reactants that are being fed (initiators, surfactants, etc....) causing coagulation. Rheology is also very important during application, because the latex is subjected to very different shear rates. For example, during coating application (e.g., brushing), the shear rate is high, but it almost decreases to zero afterwards. The pseudo-plasticity of the latex plays a critical role here because during application its viscosity should be low to reduce the energy needed to spread the coating on the substrate. Then, an intermediate viscosity is needed for leveling the coating (disappearance of the brush marks), and finally, a high viscosity is needed to avoid sagging. Therefore, knowledge of the rheology of the latexes is of utmost importance.

This has been the driving force for the development of a high number of mathematical models for predicting the rheology of concentrated dispersions. These models have been summarized by Rueda et al. [3] and will not be repeated here. When analyzing these models, it can be noticed that they mainly focus on the effect of the solid content and particle size distribution on viscosity, whereas the effect of the shear rate is not often considered. 
A model for the effect of shear rate on the viscosity of dispersions of monodispersed hard spheres was considered by Krieger and Dougherty [4]:

$\eta_{r}=\eta_{r \infty}+\frac{\eta_{r 0}-\eta_{r \infty}}{1+b \tau_{r}}$

where $\eta_{r}$ is the relative viscosity; $\eta_{r 0}$ and $\eta_{r o}$ are the relative viscosities at zero and high shear limits, respectively; $b$ is an adjustable parameter; and $\tau_{r}$ is the reduced shear stress:

$\tau_{r}=\frac{\tau a^{3}}{k_{B} T}$

where $\tau$ is the shear stress, $a$ the particle radius, $k_{B}$ is the Boltzmann constant, and $T$ is the temperature. $\eta_{r 0}$ and $\eta_{r \infty}$ depend on the volume fraction of the dispersed phase [5]

$\eta_{r j}=\left(1-\frac{\varphi}{\varphi_{n j}}\right)^{-[\eta] \varphi_{n j}} j=0, \infty$

where [ $\eta]$ is the intrinsic viscosity. $\varphi_{n 0}$ and $\varphi_{n \infty}$ are the maximum packing factors at zero and high shear, which should be experimentally determined. This model does not account for the particle size distribution.

A rheological model that included the effect of the volume fraction and shear rate on the viscosity has been proposed by Quemada [6].

$\eta=\eta_{\infty}\left(\frac{1+(\lambda \dot{\gamma})^{p}}{\left(\frac{\eta_{\infty}}{\eta_{0}}\right)^{0.5}+(\lambda \dot{\gamma})^{p}}\right)^{2}$

The viscosities at zero and high shear limits are

$\eta_{j}=\eta_{F}\left(1-0.5 k_{j} \varphi\right)^{-2} \quad j=0, \infty$

where $\eta_{F}$ is the viscosity of the dispersing fluid, $\dot{\gamma}$ is the shear rate, and $k_{o}, \mathrm{k}_{\infty}, \lambda$, and $p$ are parameters of the model, which should be estimated when either the particle size distribution or the solid content vary. This is a problem for semicontinuous emulsion polymerization, where solid content and the particle size distribution constantly vary during the process.

In the related field of filled polymeric systems (solid particles dispersed in a polymer matrix), Poslinski et al. $[7,8]$ proposed a model that accounts for the effects of the particle size distribution and shear rate. However, when the model is applied to a latex (Newtonian continuous phase with no yield stress), it reduces to Quemada's model.

On the other hand, the effect of particle interactions on the rheology is often recognized, but in most cases is not explicitly included in the model. An exception is the model proposed by Sudduth [9-12] $\ln \left(\eta_{r}\right)=[\eta] \varphi_{n}\left(\frac{1}{\sigma-1}\right)\left[\left(1-\frac{\varphi}{\varphi_{n}}\right)^{1-\sigma}-1\right]$ for $\sigma \neq 1$

where $\sigma$ is the particle-particle interaction parameter given by

$\sigma=\frac{\sigma_{P C}}{D_{1}}+\sigma_{s}$

where $\sigma_{P C}$ and $\sigma_{s}$ are adjustable parameters and $D_{1}$ is the ratio between the first and zeroth moment of the particle size distribution. The first term of the right-hand side member of Eq. 7 accounts for the fact that for a given solid content, the smaller the particles, the shorter the distance among them, and therefore, the higher the interaction.

The maximum packing fraction, $\varphi_{n}$, depends on the particle size distribution as follows

$\varphi_{n}=\varphi_{\text {nult }}-\left(\varphi_{\text {nult }}-\varphi_{m}\right) \exp \left[\alpha\left(1-\frac{D_{5}}{D_{1}}\right)\right]$

where $\varphi_{n \text {,ult }}$ is the maximum packing fraction that can be achieved with any PSD, $\varphi_{m}$ is the maximum packing fraction for monodispersed particles, and $D_{i}$ was the ratio between $i$ th and the $(i-1)$ th moments of the PSD.

Although Sudduth model describes well the dependency of the low shear viscosity with respect to the particle size distribution and solid content [13], it does not considered the effect of the shear rate.

Aravalillo et al. [14] expanded Sudduth model to include the effect of the shear rate. Sudduth model results from the integration of the following equation considering a constant particle-particle interaction parameter

$\ln \left(\eta_{r}\right)=\int_{0}^{\varphi \mathrm{final}}[\eta]\left(1-\frac{\varphi}{\varphi_{n}}\right)^{-\sigma} d \varphi$

However, Aravalillo et al. [14] argued that for commercial latexes that are stabilized by ionic and/or nonionic surfactants, the interaction parameters depend on particle-particle distance (i.e., on the solid content) and on the shear rate $[15,16]$. Furthermore, as the interaction potential of ionically [17] and sterically [18] stabilized latexes decreases exponentially with the distance between particles, the following equation of the particle-particle interaction parameter was proposed.

$\sigma=k_{1} \exp \left[-k_{2}\left(\varphi_{n}-\varphi\right)^{1 / 3} \dot{\gamma}^{k_{3}}\right]$

where $k_{1}, k_{2}$, and $k_{3}$ are adjustable parameters that account for the particle-particle interaction and are expected to depend on the emulsifier system, but to be independent of the volume fraction of the dispersed phase, the particle size distribution, and the shear rate. $k_{1}$ controls the interaction 
at very short distances when $\left(\phi_{n}-\phi\right)$ approaches zero. $k_{2}$ determines how sharp is the viscosity decrease with the dilution of latex, and $k_{3}$ describes the effect of shear rate on viscosity.

The model defined by Eqs. 9 and 10 was used to fit the rheology of four high solid content (60-68 wt \%) latexes that had different particle size distributions. The integration of Eq. 9 was carried out numerically. All the latexes were stabilized by a mixture of anionic (Dowfax 2A1) and nonionic (Disponil A3065) surfactants and contained acrylic acid. The solid content of each latex was varied by dilution. For each latex, $k_{1}, k_{2}$, and $k_{3}$ were estimated from data obtained at different solid contents and shear rates. The maximum packing fraction was calculated from the experimental particle size distribution using the Ouchiyama and Tanaka method [19]. It was found that the model fitted fairly well the experimental data and that the estimated values of $k_{2}$ and $k_{3}$ were the same for all latexes $\left(k_{2}=1.55 \times 10^{-2}\right.$ and $\left.k=0.5\right)$. On the other hand, $k_{1}$ increased with the total area of the particles and with the surface coverage, but no correlation with the composition of the surfactant mixture (ionic/nonionic ratio) was observed. This was attributed to the high amount of acrylic acid used in the formulation of these latexes that with the contribution of the sulfate groups from the initiator and that of the ionic surfactant masked the effect of the nonionic surfactant. In other words, the particle-particle interaction was mainly electrostatic. Therefore, one wonders about the ability of the model to describe the rheology of latexes stabilized with nonionic surfactants and the dependency of the parameters of the model on the characteristics of the surfactant.

This article attempts to clarify these points. Latexes stabilized with different nonionic surfactants (polyvinyl alcohol, PVOH) and Disponil A3065) were synthesized, and the model was used to fit experimental results where the volume fraction of the dispersed phase, the particle size distribution, the temperature, and the shear rate were varied.

\section{Experimental}

Table 1 summarizes the high-solid content latexes employed in this work. These waterborne polymeric dispersions were produced via miniemulsion copolymerization of vinyl acetate and VeoVa10 ${ }^{\circledR}$ (Hexion trade mark). Latexes $L_{\text {exp1- }}{ }^{-}$ $L_{\text {exp3 }}$ were stabilized by polyvinyl alcohol (Celvol 205 kindly supplied by Wacker Chemie, degree of hydrolysis $=87-89 \%$; weight average molecular weight $=31,000-50,000 \mathrm{~g} / \mathrm{mol}$ ). Latex $L_{\text {exp4 }}$ was prepared with Disponil A3065 (supplied by Cognis, $65 \mathrm{wt} \%$ active substance). The hairy layer formed by Disponil A3065 was expected to be thinner than that of $\mathrm{PVOH}$, which would allow to shed light on the effect of the type of non-ionic surfactant on the parameters of the model. Latexes $L_{\exp 1}$ and $L_{\text {exp2 }}$ were synthesized by semicontinuous miniemulsion polymerization at $65{ }^{\circ} \mathrm{C}$ using sodium persulfate as initiator and a feeding time of $4 \mathrm{~h}$. After the feeding period, the system was allowed to react for $60 \mathrm{~min}$ at $65{ }^{\circ} \mathrm{C}$. In latex $L_{\text {exp } 1}, 2 \mathrm{wt} \%$ of styryl acrylate (SA) was used to reinforce the stability of the miniemulsion against Oswald ripening [20]. Latex $L_{\exp 3}$ was prepared by batch miniemulsion polymerization of a 50/50-wt/wt mixture of the miniemulsions used to synthesize latexes $L_{\exp 1}$ and $L_{\exp 2}$. Latex $L_{\text {exp } 4}$ was prepared using the same procedure than for latex $L_{\text {exp }}$, but using Disponil A3065 instead of PVOH.

Figure 1 shows the normalized number $\left(n *\left(d_{p}\right)\right)$ and weight $\left(w^{*}\left(d_{p}\right)\right)$ PSDs of the latexes employed, as measured by the DCP-Centrifuge. The normalizations were as follows:

$$
\begin{aligned}
& n *\left(d_{p}\right)=\frac{n\left(d_{p}\right)}{\int_{0}^{\infty} n\left(d_{p}\right) d\left(d_{p}\right)} \\
& w *\left(d_{p}\right)=\frac{d_{p}^{3} n\left(d_{p}\right)}{\int_{0}^{\infty} d_{p}^{3} n\left(d_{p}\right) d\left(d_{p}\right)}
\end{aligned}
$$

Table 1 shows that solid content of latexes were similar and that the concentration of emulsifier was the same. However, the shape of the PSDs and the total surface area of the latexes were different (Fig. 1). Latex $L_{\exp 1}$ was a bimodal dispersion with the lowest number of particles $(\mathrm{Np})$ and the highest particle diameter $\left(d_{p}\right)$, which was a product of the particle formation by droplet nucleation and conventional nucleation mechanisms (heterogeneous or homogeneous). The reason of this dual nucleation process was the poor stability of the miniemulsion against Oswald ripening. In latex $L_{\text {exp2 }}$, the miniemulsion was stable due to the presence of $\mathrm{SA}$ and this led to a monomodal dispersion with a lower $d_{p}$
Table 1 Latexes employed in the experiments $\left({ }^{\mathrm{a}} \mathrm{phm}=\right.$ weight parts per hundred monomer)

\begin{tabular}{lllllll}
\hline Latex & Solid content & Volume fraction & Emulsifier system ${ }^{\mathrm{a}}$ & $\boldsymbol{d}_{\boldsymbol{p}}(\mathbf{n m})$ & PDI & $\begin{array}{l}\text { Np } \\
(\mathbf{p a r t} / \mathbf{L})\end{array}$ \\
\hline$L_{\text {exp1 }}$ & 0.49 & 0.451 & Celvol 205 $(8 \mathrm{phm})$ & 512 & 1.590 & $3.90 \times 10^{15}$ \\
$L_{\exp 2}$ & 0.50 & 0.460 & Celvol 205 $(8 \mathrm{phm})$ & 361 & 1.406 & $1.74 \times 10^{16}$ \\
$L_{\exp 3}$ & 0.52 & 0.476 & Celvol 205 $(8 \mathrm{phm})$ & 308 & 1.796 & $2.58 \times 10^{16}$ \\
$L_{\exp 4}$ & 0.51 & 0.468 & Disp A3065 $(8 \mathrm{phm})$ & 393 & 1.828 & $1.39 \times 10^{16}$ \\
\hline
\end{tabular}


Fig. 1 Number and weight PSDs of polymeric dispersions
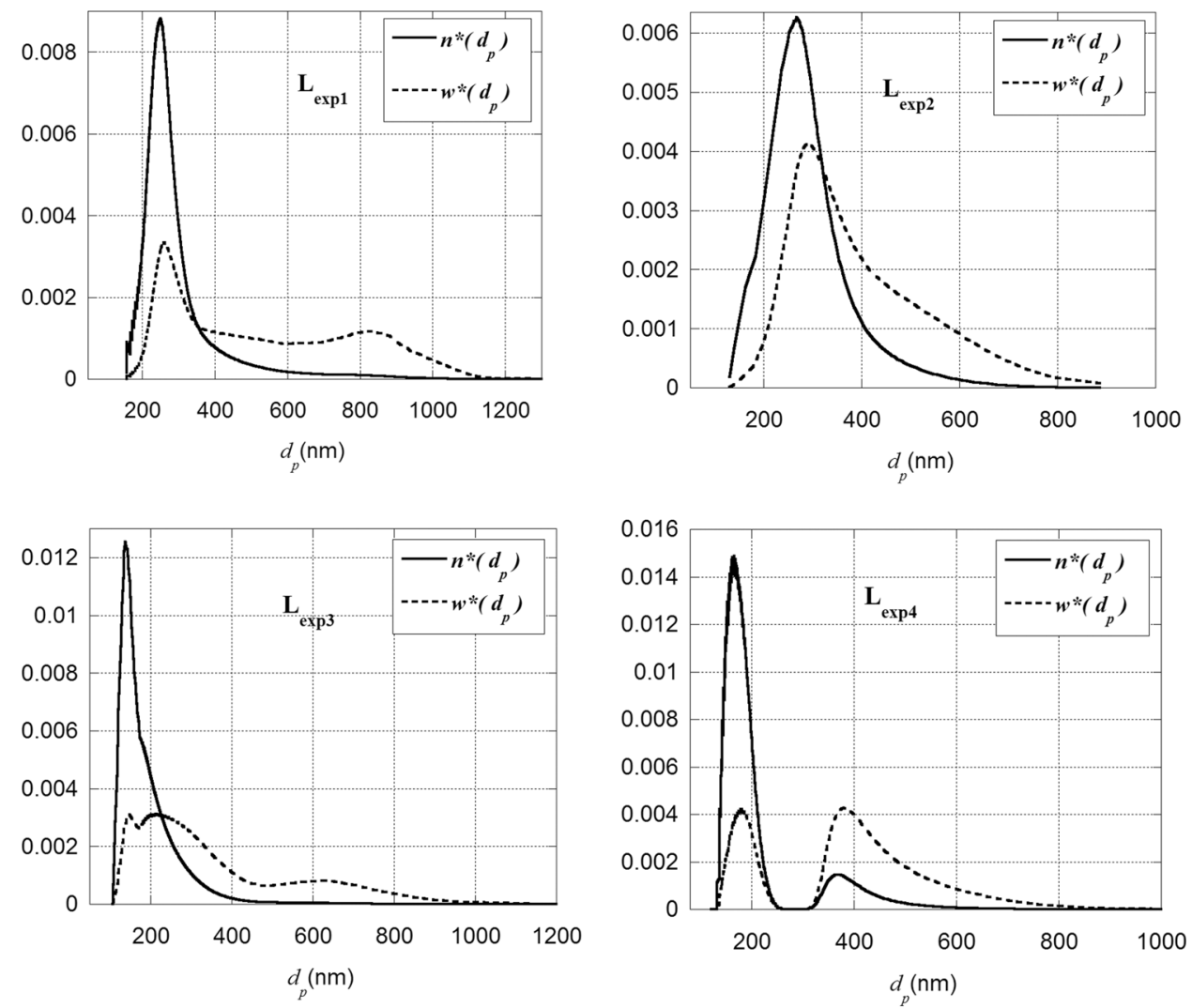

(higher $\mathrm{Np}$ as compared to $L_{\mathrm{exp} 1}$ ), which was a product of predominant droplet nucleation. Latex $L_{\text {exp3 }}$ was prepared by batch polymerization of the mixture of miniemulsions leading to a latex with the highest polydispersity (among the poly(vinyl alcohol) stabilized latexes). This was the sample with the highest $\mathrm{Np}$ and the lowest $d_{p}$ which means the highest surface area. In the case of $L_{\text {exp4 }}$, a bimodal PSD (two peaks with similar intensities more apparent in the weight PSD) was obtained. Similar to latex $L_{\exp 1}$, this was the result of dual nucleation mechanisms.

In order to obtain different solid contents, the original latexes were diluted and concentrated by adding and evaporating water, respectively. The dilution with water caused a modification on the surface concentration of surfactant, because some surfactant migrated to water phase. However, it is believed that this was not significant; because the dilution was not substantial (the lowest solid content was 40 $\mathrm{wt} \%$ ). To concentrate the dispersion, approximately $100 \mathrm{~g}$ of the latex were placed in a 250-ml glass vessel and heated in a bath water at $40{ }^{\circ} \mathrm{C}$; magnetic agitation was provided during the process. To check the integrity of the original characteristics of the latex after the concentration, the particle size distribution was measured and no change was observed.

The viscosity of the latexes was measured with a stress rheometer Haake model VT500 that is fitted with concentric cylinders, and different sensors (according to the solid content viscosity range) were used to perform the analysis. SV and MVDIN sensors were employed to measure the samples with 50-60 wt $\%$ and $45 \mathrm{wt} \%$ solid content, respectively. Temperature was varied from 15 to $35^{\circ} \mathrm{C}$.

In latexes stabilized with short molar mass surfactants (e.g., Disponil 3065), the viscosity of the continuous phase (serum) can be approximated to $1 \mathrm{mPa}$ (water viscosity) due to the low molecular weight of these surfactants. This is not the case when using PVOH. Therefore, the serum was separated from the dispersion and its viscosity measured. Approximately $30 \mathrm{ml}$ of latex were placed in thick wall polycarbonate centrifuge tubes at $40,000 \mathrm{rpm}$. Serum was carefully decanted, and the viscosity was measured with a concentric cylinder viscosimeter (UK ELV-8) using a spindle no. 1 at 30 r.p.m. The temperature was fixed with a bath water at $25^{\circ} \mathrm{C}$. Table 2 shows the results obtained. It can be seen that serum viscosity increased with solid content.

\section{Results and discussion}

For each latexes, the parameters of the model were estimated by minimizing the differences between experimental data in which $\dot{\gamma}$ and $\varphi$ were varied and model prediction by means of the direct search algorithm of Nelder and Mead [21]. It was found that for all the latexes, the values of $k_{2}$ and $k_{3}$ were 
Table 2 Effect of solid content on latex serum

\begin{tabular}{llll}
\hline Dispersion $\boldsymbol{L}_{\text {exp1 }}$ & & & \\
Solid content & $45.0 \%$ & $48.5 \%$ & $53.5 \%$ \\
Serum viscosity (mPa s) & 5.0 & 5.3 & 6.3 \\
Dispersion $\boldsymbol{L}_{\text {exp2 }}$ & & & \\
Solid content & $45.0 \%$ & $50.0 \%$ & $55.0 \%$ \\
Serum viscosity (mPa s) & 2.0 & 3.3 & 4.3 \\
Dispersion $\boldsymbol{L}_{\text {exp3 }}$ & $40.2 \%$ & $51.3 \%$ & $57.2 \%$ \\
Solid content & 4.0 & 5.0 & 6.3 \\
\hline Serum viscosity (mPa s) & & \\
\hline
\end{tabular}

close to $1.0 \times 10^{-2}$ and 0.45 , respectively. Therefore, these values were fixed and only $k_{1}$ was estimated.

Figure 2 compares experimental results with model predictions for all the latexes. In these experiments, the solid content and the shear rate were varied for each dispersion. The estimated values of the parameter $k_{1}$ are given in Table 3. Figure 2 shows that a model fitted well the experimental data for both the polymeric $(\mathrm{PVOH})$ and the nonionic (Disponil A3065) surfactants, which shows that the model can also describe the rheology of the latexes stabilized with non-ionic surfactants.

Comparing latexes stabilized with $\mathrm{PVOH}$, it can be noticed that $k_{1}$ increased from $L_{\exp 1}$ to $L_{\exp 3}$. In addition, latex $L_{\exp 4}$ that was stabilized with the small molecular weight Disponil A3065 presented the lowest value of $k_{1}$, which indicated a lower particle-particle interaction. Figure 3 shows that $k_{1}$ increased with the total surface of the
Table 3 Estimated values of $k_{1}$ $\left(k_{2}=1.0 \times 10^{-2}\right.$ and $\left.k_{3}=0.45\right)$

\begin{tabular}{ll}
\hline Dispersion & $\boldsymbol{k}_{\mathbf{1}}$ \\
\hline$L_{\text {exp1 }}$ & 2.34 \\
$L_{\text {exp2 }}$ & 2.58 \\
$L_{\text {exp3 }}$ & 2.78 \\
$L_{\text {exp4 }}$ & 2.04 \\
\hline
\end{tabular}

particles, likely because of the higher the $\mathrm{Np}$, the closer the packing, and consequently, the interactions among particles increased.

In order to study the effect of surface coverage and surfactant concentration on $k_{1}$, different amounts of $\mathrm{PVOH}$ were added to latex $L_{\exp 1}$. The main idea was to maintain a constant surface area while measuring the effect of increasing the PVOH on the rheological behavior. Figure 4a shows that the viscosity increased with the concentration of PVOH in latex. The increase was stronger than the variation of the viscosity of the serum $(8 \mathrm{phm}=5.3 \mathrm{mPa} \mathrm{s}, 12 \mathrm{phm}=6.1 \mathrm{mPa} \mathrm{s}$, $16 \mathrm{phm}=7.3 \mathrm{mPa} \mathrm{s}, 20 \mathrm{phm}=9.5 \mathrm{mPa}$.) which shows that it was mainly due to stronger particle-particle interactions. The experimental data were fitted with the model, and a good agreement between model results and experimental data was obtained. It was found that $k_{1}$ increased with the surface coverage (Fig. 4b).

The results showed in the previous section confirmed that the model is able to describe the effect of particle size distribution, volume fraction, surfactant concentration, and shear rate on the viscosity of water-borne dispersions stabilized by non-ionic surfactants and polymeric stabilizers.
Fig. 2 Experimental data (square) versus model predictions (line) for all the latexes
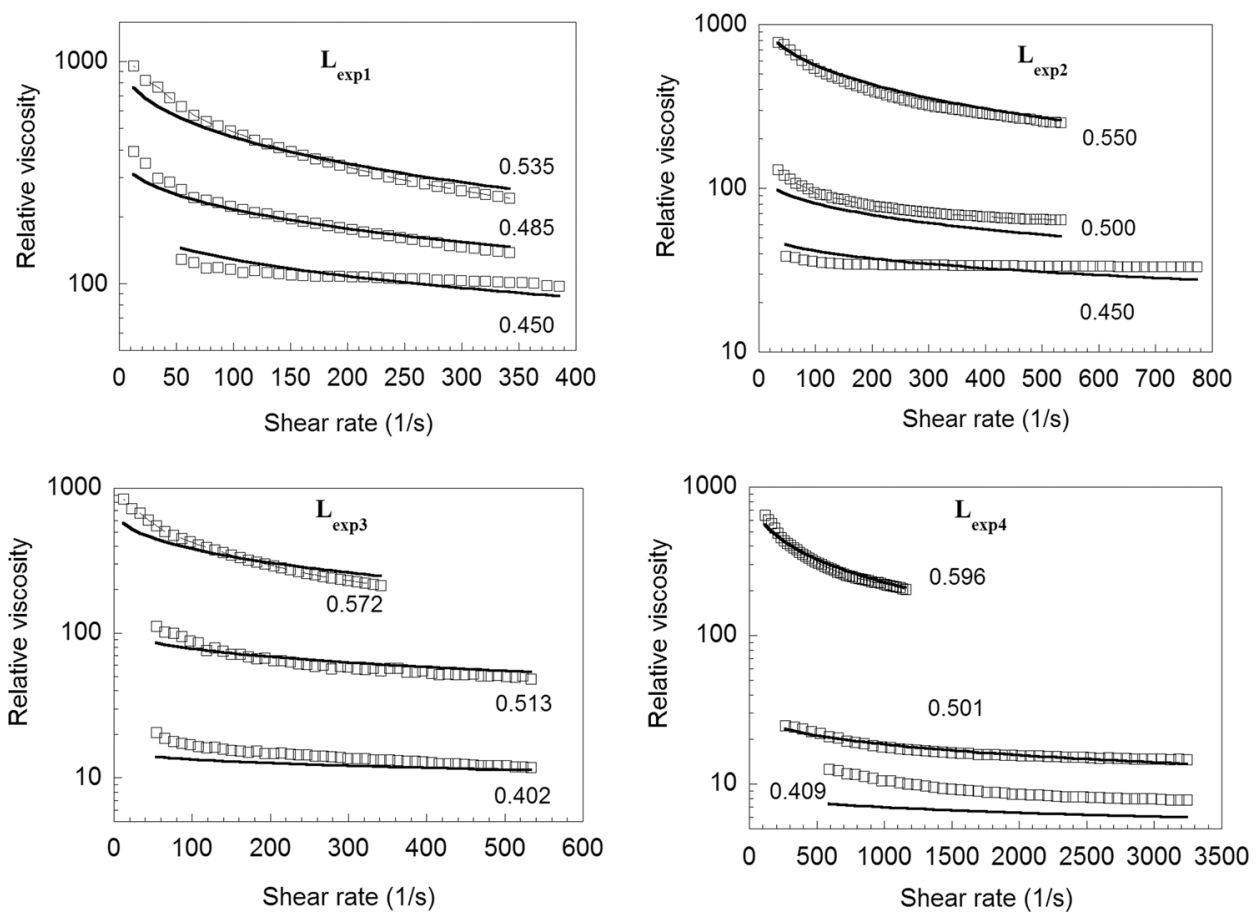


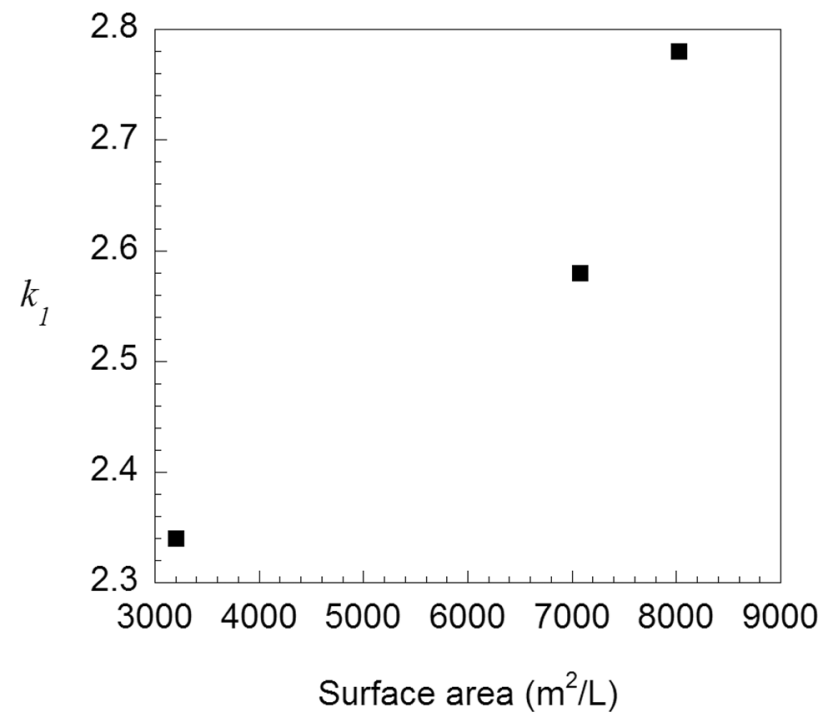

Fig. 3 Effect of the total surface area of the particles on $k_{1}$

Nevertheless, there is another important variable to study, and this is the temperature. Reactors and mixing tanks can be operated at different temperatures, and this could cause variations in the viscosity of the dispersions. Therefore, in this section, the effect of the temperature on the latex viscosity was assessed.

The viscosity of dispersions $L_{\exp 1}$ and $L_{\exp 2}$ was measured at different temperatures. The serum was separated by means of centrifugation techniques and its viscosity measured at different temperatures. The results in Table 4 show that the viscosity of the serum decreased with temperature. It has been shown that the viscosity of aqueous solutions of poly(ethylene oxide) (PEO) is closely related to the extent of hydrogen bonding between PEO chains and water molecules $[22,23]$. The extent of hydrogen bonding decreased with an increase in temperature; thus, the viscosity of PEO aqueous solutions decreased. For $\mathrm{PVOH}$, this situation is more complicated since there is a strong inter and intra chain hydrogen bonding formed between polar hydroxyl groups in the PVOH molecules [24-27]. The extent of both inter and intra chain hydrogen bonding and solute-solvent hydrogen
Table 4 Effect of temperature on serum viscosity (both dispersions had $45 \mathrm{wt} \%$ solid content)

\begin{tabular}{llll}
\hline Dispersion $\boldsymbol{L}_{\text {exp1 }}$ & & & \\
Temperature $\left({ }^{\circ} \mathrm{C}\right)$ & 15 & 25 & 35 \\
Serum viscosity (mPa s) & 6 & 5 & 4 \\
Dispersion $\boldsymbol{L}_{\text {exp2 }}$ & & & \\
Temperature $\left({ }^{\circ} \mathrm{C}\right)$ & 15 & 25 & 35 \\
Serum viscosity $(\mathrm{mPa} \mathrm{s})$ & 3 & 2 & 1 \\
\hline
\end{tabular}

bonding is determined by the degree of hydrolysis. PVOH grades with higher degree of hydrolysis show higher viscosity and lower water solubility due to gel formation and to increased inter and intra bonding, respectively. On the other hand, at low degree of hydrolysis, the increase of acetate content led to an increase on viscosity and to a decrease in water solubility. The PVOH grade employed to produce the latexes used in this work presents a medium degree of hydrolysis (87-89\%). Briscoe et al. [27] showed that PVOH grades with a medium hydrolysis level present a viscosity behavior with temperature similar to PEO molecules, e.g., the viscosity decreased with an increase in temperature.

Figure 5 shows that the viscosity decreased as temperature increased and that the model captured well the effect of the temperature on the dispersion viscosity. Finally, $k_{1}$ decreased as temperature increased (Fig. 6), suggesting a weaker interaction among particles. The reduction of the hydrogen bonding with temperature resulted in less extended hairy layer and therefore in lower particle-particle interactions.

These results validated the model showing its potential for accounting for the effect of the volume fraction, the particle size distribution, the shear rate, and temperature on the viscosity of nonionically stabilized latexes. The combination of these findings with the previously published results on the rheology of anionically stabilized latexes [14] demonstrates that the model is able to describe the rheology of any type of latexes. Therefore, the model proposed is a powerful tool for designing polymerization strategies aiming at producing latexes with high solid content and moderate viscosity, avoiding the use of trial and error methods.
Fig. 4 Effect of surface coverage on a viscosity (model (line) and experimental data (symbols) and $\mathbf{b} k_{1}$
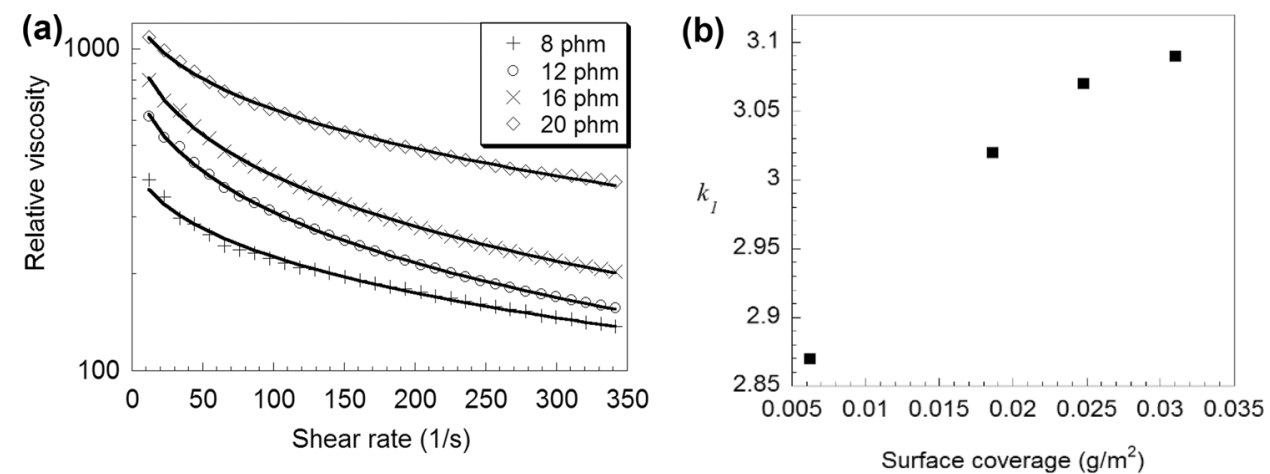
Fig. 5 Experimental data (square) versus model predictions (line) for the sample dispersions describing the effect of the temperature $\left(k_{2}=1.0 \times 10^{-2}\right.$ and $k_{3}=0.45$ )
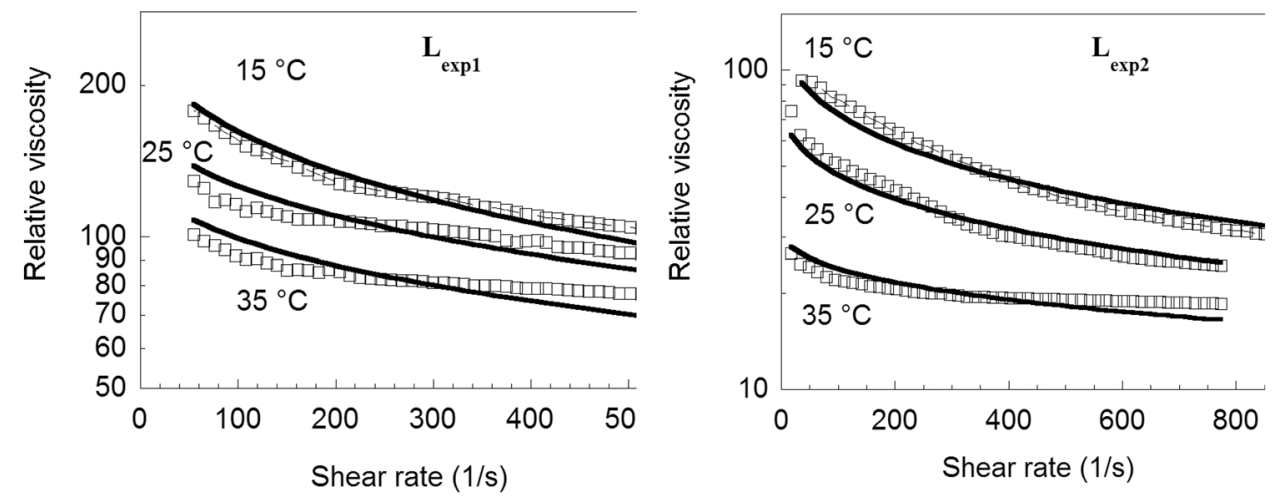

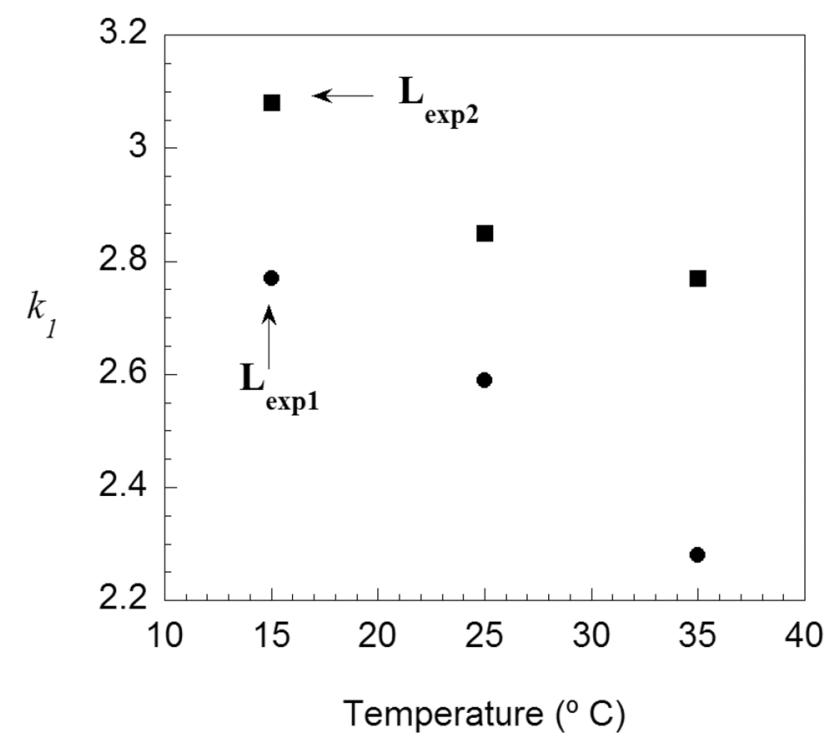

Fig. 6 Effect of temperature on $k_{1}$

\section{Conclusions}

In the present work, the performance of a rheology model that accounts for the effects of the volume fraction of the dispersed phase, the particle size distribution, and the shear rate on the viscosity of latexes stabilized with non-ionic surfactants and polymeric stabilizers was presented.

The model was validated by fitting viscosity data obtained at varying shear rates with several high solid content latexes stabilized with both high molecular weight polymeric emulsifier $(\mathrm{PVOH})$ and nonionic low molecular weight emulsifier (Disponil A3065). The dispersions had widely different PSDs, and solid contents ranging from 40.2 to $59.6 \mathrm{wt} \%$. The model fitted well the experimental data.

The model contains three parameters $\left(k_{1}, k_{2}\right.$, and $\left.k_{3}\right)$ that account for the interaction among particles. $k_{1}$ accounts for the interaction at very short distances, $k_{2}$ determines how fast the interaction decreases with the distance between particles, and $k_{3}$ accounts for the effect of the shear rate on the interaction.
The parameters were independent of the volume fraction, the particle size distribution, and the shear rate. Under the experimental conditions employed, $k_{2}$ and $k_{3}$ were not influenced by the surfactant type and concentration. On the other hand, it was found that the use of polymeric surfactants led to increased particle-particle interactions because $k_{1}$ was higher in the case of $\mathrm{PVOH}$ stabilized latexes compared to the $k_{1}$ obtained for the latex stabilized with a nonionic low molecular weight surfactant (Disponil A3065). The parameter $k_{1}$ increased with an increase of the total particle surface area and with an increase of the emulsifier concentration (surface coverage) for a constant surface area of the particles.

In addition, the model was able to describe the effect of the temperature on the PVOH stabilized dispersion viscosity. A decrease of the temperature led to an increase of the latex viscosity. $k_{1}$ increased with an increase in temperature, while $k_{2}$ and $k_{3}$ were no affected by this variable.

The combination with previously reported results [14] shows that the model can describe the rheology of any type of latexes estimating a small number of parameters. This opens the possibility of designing polymerization strategies aiming at achieving high-solid content-low viscosity latexes.

Funding Open Access funding provided thanks to the CRUE-CSIC agreement with Springer Nature. This study was supported by Ministerio de Ciencia e Innovación (PID2019-107889GA-I00).

\section{Declarations}

Conflict of interest The authors declare no competing interests.

Open Access This article is licensed under a Creative Commons Attribution 4.0 International License, which permits use, sharing, adaptation, distribution and reproduction in any medium or format, as long as you give appropriate credit to the original author(s) and the source, provide a link to the Creative Commons licence, and indicate if changes were made. The images or other third party material in this article are included in the article's Creative Commons licence, unless indicated otherwise in a credit line to the material. If material is not included in the article's Creative Commons licence and your intended use is not permitted by statutory regulation or exceeds the permitted use, you will 
need to obtain permission directly from the copyright holder. To view a copy of this licence, visit http://creativecommons.org/licenses/by/4.0/.

\section{References}

1. Urban D, Takamura K (2002) Polymer Dispersions and Their Industrial Applications. Wiley-VCH

2. Guyot A, Chu F, Schneider M, Graillant C, McKenna TF (2003) High solids content latexes. Prog Polym Sci 27:1573-1615. https://doi.org/10.1016/S0079-6700(02)00014-X

3. Rueda MM, Auscher MC, Fulchiron R, Périe T, Martin G, Sonntag P, Cassagnau P (2017) Rheology and applications of highly filled polymers : A review of current understanding. Prog Polym Sci 66:22-53. https://doi.org/10.1016/j.progpolymsci.2016.12.007

4. Krieger IM, Dougherty TJA (1959) A mechanism for no-Newtonian flow in suspensions of rigid spheres. Trans Soc Rheol 3:137-152. https://doi.org/10.1122/1.548848

5. Krieger IM (1972) Rheology of monodisperse latices. Adv Colloid Interface Sci 3:111-136. https://doi.org/10.1016/0001-8686(72) 80001-0

6. Quemada D, Flaud P, Jezequel PH (1985) Rheological properties and flow of concentrated dispersed media I-Modelling of steady and unsteady behavior. Chem Eng Commun 32:61-83. https://doi. org/10.1080/00986448508911642

7. Poslinski AJ, Ryan ME, Gupta RK, Sesshari, SG Frechette FJ (1988) Rheological behaviour of filled polymeric systems I. Yield stress and shear-thinning effects. J Rheol 32:703-735. https://doi. org/10.1122/1.549987

8. Poslinski AJ, Ryan RME, Gupta RK, Sesshari SG, Frechette FJ (1988) Rheological behaviour of filled polymeric systems II. The effect of a bimodal size distribution of particulates. J Rheol 32:751-771. https://doi.org/10.1122/1.549991

9. Sudduth RD (1993) A generalized model to predict the viscosity of solutions with suspended particles I. J Appl Polym Sci 48:2536. https://doi.org/10.1002/app.1993.070480104

10. Sudduth RD (1993) A new method to predict the maximum packing fraction and the viscosity of solutions with a size distribution of suspended particles II. J Appl Polym Sci 48:37-55. https://doi. org/10.1002/app.1993.070480105

11. Sudduth RD (1993) A generalized model to predict the viscosity of solutions with suspended particles. III. Effects of particle interaction and particle size distribution. J Appl Polym Sci 50:123147. https://doi.org/10.1002/app.1993.070500115

12. Sudduth RD (1994) A generalized model to predict the viscosity of solutions with suspended particles. IV. Determination of optimum particle-by-particle volume fractions. J Appl Polym Sci 52:985-996. https://doi.org/10.1002/app.1994.070520717

13. do Amaral M, van Es S, Asua JM (2004) Effect of the particle size distribution on the low shear viscosity of high-solids content latexes. J Polym Sci Part A: Polym Chem 42:3936-3946. https:// doi.org/10.1002/pola.20269
14. Arevalillo A, do Amaral M, Asua JM (2006) Rheology of concentrated polymeric dispersions. Ind Eng Chem Res 45:3280-3286. https://doi.org/10.1021/ie0510371

15. Mewis J, Frith WJ, Strivens TA, Russel WB (1989) The rheology of suspensions containing polymerically stabilized particles. AIChE J 35:415-422. https://doi.org/10.1002/aic.690350309

16. Buscall R (1991) Effect of long-range repulsive forces on the viscosity of concentrated lattices: Comparison of experimental data with an effective hard-sphere model. J Chem Soc Faraday Trans 87:1365-1370. https://doi.org/10.1039/FT9918701365

17. Hunter R (1992) Foundations of colloid science. Clarendon Press, Oxford

18. Israelachvili J (1997) Intermolecular and Surface Forces-3rd Edition. Academic Press, London

19. Ouchiyama N, Tanaka T (1984) Porosity estimation for random packing of spherical particles. Ind Eng Chem Fundam 23:490 493. https://doi.org/10.1021/i100016a019

20. Asua JM (2018) Ostwald ripening of reactive costabilizers in miniemulsion polymerization. Eur Polym J 106:30-41. https:// doi.org/10.1016/j.eurpolymj.2018.07.001

21. Nelder JA, Mead R (1965) A simplex method for function minimization. Computer J 7:308-313. https://doi.org/10.1093/comjnl/ 7.4.308

22. Briscoe B, Luckham P, Zhu S (1996) Rheological study of poly(ethylene oxide) in aqueous salt solutions at high temperature and pressure. Macromolecules 29:6208-6211. https://doi.org/10. 1021/ma960667z

23. Briscoe B, Luckham P, Zhu S (1998) Rheological properties of poly(ethylene oxide) aqueous solutions. J Appl Polym Sci 70:419_ 429. https://doi.org/10.1002/(SICI)1097-4628(19981017)70:3\% 3C419::AID-APP1\%3E3.0.CO;2-Q

24. Finch C (1973) Poly(vinyl alcohol), Properties and Developments. Wiley, New York

25. Shibayama M, Uesake M, Inamoto S, Mihara H, Nombra S (1996) Analogy between swelling of gels and intrinsic viscosity of polymer solutions for ion-complexed poly(vinyl alcohol) in aqueous medium. Macromolecules 29:885-891. https://doi.org/10.1021/ ma951194j

26. Maeda H, Kawai T, Sekii S (1959) Intra- and intermolecular hydrogen bonds in poly(vinyl alcohol) solutions. J Polym Sci 35:288-292. https://doi.org/10.1002/pol.1959.1203512831

27. Briscoe B, Luckham P, Zhu S (2000) The effects of hydrogen bonding upon the viscosity of aqueous poly(vinyl alcohol) solutions. Polymer 41:3851-3860. https://doi.org/10.1016/S00323861(99)00550-9

Publisher's Note Springer Nature remains neutral with regard to jurisdictional claims in published maps and institutional affiliations. 